\title{
AUTOMATIC SEGMENTATION OF INTERNAL STRUCTURES OF THE BRAIN IN MR IMAGES USING A TANDEM OF AFFINE AND NON RIGID REGISTRATION OF AN ANATOMICAL BRAIN ATLAS
}

\author{
Meritxell Bach Cuadra, Olivier Cuisenaire, Reto Meuli* and Jean-Philippe Thiran \\ Swiss Federal Institute of Technology (EPFL) \\ Signal Processing Laboratory (LTS) \\ CH-1015 Lausanne, Switzerland \\ WWW home page: http://ltswww.epfl.ch/ brain \\ \{meritxell.bach,Olivier.Cuisenaire,JP.Thiran\}@epfl.ch \\ * Department of Radiology \\ Lausanne University Hospital (CHUV) \\ CH-1011 Lausanne, Switzerland
}

\begin{abstract}
In the study of many neurological pathologies, the accurate quantization of the white matter (WM) and gray matter (GM) volumes of the brain is essential. Moreover, regional volume calculations may bring even more useful diagnostic information. In this paper, we present therefore the segmentation of internal structures of the brain for further regional WM and GM volume quantization. A priori information about the brain anatomy is included in the segmentation process by the registration of the patient MR images with a computerized brain atlas. We propose here the combination of a global affine transformation used to initialize key boundary surfaces (lateral ventricles and cortical surfaces) of both images with a local free-form transformation based on an optical flow algorithm. We apply this technique to segment the cerebellum and the cerebral trunk in order to exclude them from our WM and GM volume quantization. Validation has been conducted on a large number of images, showing excellent results.
\end{abstract}

\section{INTRODUCTION}

The accurate analysis of internal structures of the brain is undoubtedly of great interest for the study and the treatment of various pathologies. Among them, the quantization of gray and white matter volumes may be of major interest in neurodegenerative disorders such as Alzheimer disease, in movements disorders such as Parkinson or Parkinson related syndrome, in white matter metabolic or inflammatory disease, in congenital brain malformations or perinatal brain damage, or in post traumatic syndrome. In such cases, it is necessary to consider only the volume of white and gray matter in specific regions of the brain such as hemispheric lobes or individual deep gray matter nucleus, excluding other regions like the cerebral trunk and the cerebellum. It is therefore essential to accurately segment the brain in order to detect the regions of interest for the volumetric quantization. As it is important for this process to be robust, inclusion of a priori information about the task to be carried out is necessary. In this work we study the use of an atlas registration technique to segment the brain structures. The process consists in finding the transformation that deforms one image called deformable model where the structures of interest have already been defined (i.e. an electronic atlas of the brain) toward another image called reference image where structures of interest will be projected (i.e. the patient images). Both local and global transformations are considered. The global transformation used here is an affine registration. However, this technique is not enough to achieve a good local registration. In this case our solution is to combine both global affine and local non-rigid transformations. The studies in the non-rigid techniques based on a complete $3 \mathrm{D}$ deformable model have received a lot of interest recently, ranging from the elastic model approach (Bajcsy [1]) to viscous fluid models (Christensen [2], BroNielsen [3]). Another interesting approach is the demonbased method of Thirion [4] that presents a different point of view as compared to existing non-rigid attractor-based techniques. In this work we have bet on an affine registration with a non-rigid matching using the demons algorithm of Thirion. This methodology follows the same idea as Hartmann et al. in [5]. Applying first the affine registration we obtain a very good initialization to apply the demons algo- 

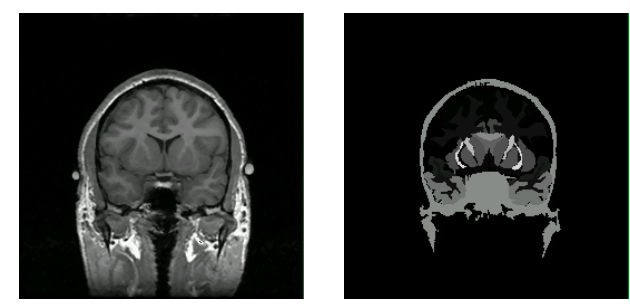

Fig. 1. MR and labeled coronal image of the Surgical Planning Laboratory (SPL) atlas.

rithm avoiding large initial differences between deformable and reference images. Then, the fast fully automatic demon method is used to compensate the morphological and morphometrical differences between internal structures of the brain. We perfomed visual validation. When the registration of the atlas and the patient image is completed, it is easy to extract the region of interest, simply by considering only regions of the brain included in the desired region of the registered atlas. Finally, thanks to previous works of our group, an algorithm based on a robust parameter estimation of mixtures using genetic algorithms is used to calculate the WM and GM volumes in the regions of interest [6].

This paper is structured as follows. In Section 2 we briefly describe the data and three methods used in this application. Next, in Section 3, we present and analyze typical results of two of our studied patients. Conclusions on the performance and insight on future work are discussed in Section 4.

\section{MATERIAL AND METHODS}

\subsection{Atlas and patient data}

The digital atlas used for this application is the one from the Surgical Planning Laboratory (SPL) of Harvard Medical School [7]. This atlas is made of MR data from a single normal subject scanned with high resolution $(256 \times 256 \times$ 160 volume data set in coronal orientation with $0.9375 \times$ $0.9375 \times 1.5$ voxel size). Data information is presented by both the MRI data set and the corresponding label map of the segmented structures of the brain (see Figure 1). The patient data sets are formed by T2-weighted MR images of healthy subjects $(256 \times 256 \times 124$ volume data set in sagittal orientation with $1 \times 1 \times 1.25$ voxel size).

\subsection{Global affine transformation}

The global transformation used here is an affine transformation whose coefficients minimize a distance measure of the atlas to the correspondent structures in the target image. In this case, the registration method is based on the matching of 2 important anatomical landmarks: the cortical surface and the ventricular system. A complete description of the method is presented in [8].

\subsection{Non-rigid transformation}

The local non-rigid deformation used in this work follows the demons algorithm proposed by Thirion [4]. Many types of transformations can be used within demons framework. Our choice here is a totally free-form deformation represented by a 3D floating point vector for each voxel. For the resampling algorithm we use a tri-linear interpolation. There are several types of demons but their general characteristic is its binary behavior (inside / outside) to decide pushing direction. The starting point to calculate demons force is the concept of instantaneous optical flow equation as presented in [9]. The hypothesis is that there is a conservation of the intensity in time of the points under motion:

$$
i(x(t), y(t), z(t), t)=\text { const in time. }
$$

If we take derivatives:

$$
\frac{\partial i}{\partial x} \frac{\partial x}{\partial t}+\frac{\partial i}{\partial y} \frac{\partial y}{\partial t}+\frac{\partial i}{\partial z} \frac{\partial z}{\partial t}=-\frac{\partial i}{\partial t} .
$$

In the case of having two volume images to register, $f$ (reference image) and $g$ (deformable model), we consider that these images are two consecutive frames and we are interested in $\vec{v}$, the motion field that brings $g$ closer to $f$. Thus, we consider that $\frac{\partial i}{\partial t}=f-g$, and that $\vec{v}=\left(\frac{\partial x}{\partial t}, \frac{\partial y}{\partial t}, \frac{\partial z}{\partial t}\right)$ is the instantaneous velocity from $g$ to $f$. So,

$$
\vec{v} \cdot \vec{\nabla} f=g-f .
$$

Since this equation does not suffice to determine $\vec{v}$ locally we regularize the problem by the assumption that the motion has locally the same direction as the spatial gradient. That leads to the following equation for the local motion:

$$
\vec{v}=\frac{(g-f) \vec{\nabla} f}{\vec{\nabla} f^{2}} .
$$

However, this equation is not stable if the gradient norm of the reference image is small. To avoid this possible discontinuity in (4) we use a modified version of the demon force:

$$
\vec{p}=\frac{(g-f) \vec{\nabla} f}{\vec{\nabla} f^{2}+(g-f)^{2}} .
$$

The demon algorithm is implemented in an iterative way that follows these steps:

1. Pre-compute demon points.

2. Compute, for each demon point, the displacement vector generating a local force field between the actual deformable model and the reference image. 

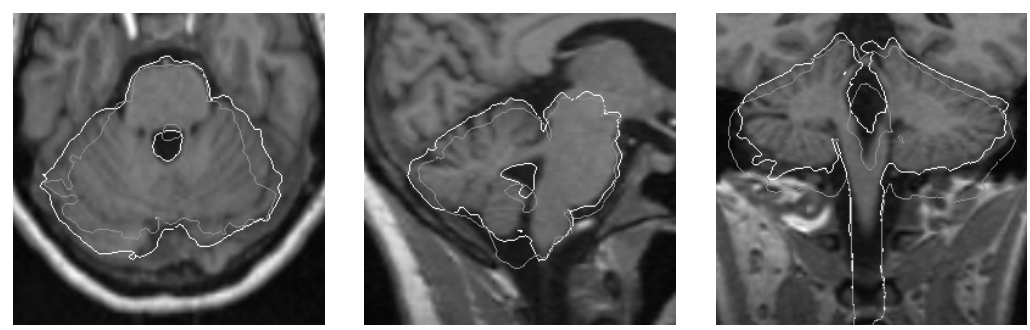

Fig. 2. Contours of original (in gray) and registered (in white) object of the atlas (cerebellum) for the first patient. It can been seen as white contour matches much better the cerebellum that gray one even if sometimes there is an initial large difference.
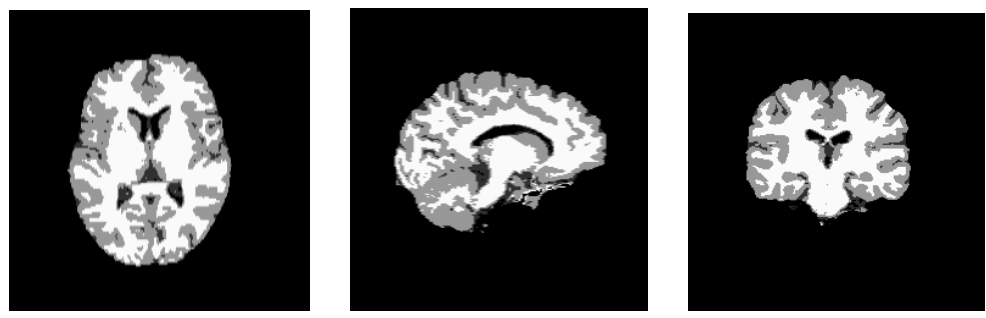

Fig. 3. Final classification of gray and white matter.

3. Filter these forces by a Gaussian filter.

4. Compute the new global transformation.

5. Go to step 2 .

Furthermore, the algorithm has been implemented in a bijectivity way [4]. Both direct and inverse transformations are computed at each iteration and also the residual deformation. Then, they are equally compensated by a half of this residual deformation to maintain both transformations compatible each other. Although this method requires more than twice the one way computation, it has the advantage that provides at the end not only the direct transformation, but also an inverse one. Another known advantage of this method is its robustness in preserving the anatomical coherence of the images.

Moreover, the algorithm is implemented in a multiscale way, increasing the speed, the robustness and the convergence of the registration.

\subsection{Gray and white matter volume quantization}

The head is constituted of different types of tissues. The correspondent gray values in a MR image can be modeled by a finite mixture of multivariate Gaussian distributions. Schroeter et al. [6] have presented two methods for a robust estimation of the statistics of the desired tissues even if they are mixed with other data of less interest. Their algorithm follows the next steps:
1. Selection of images where brain is well represented.

2. Calculation of the histogram and computation of background threshold.

3. Determination of a mask of the brain using mathematical morphological operations.

4. Estimation of GM and WM statistics using a genetic algorithm applied on the histogram.

\section{RESULTS}

In this section some figures are presented to illustrate the results that we have obtained. Even if we have obtained good results for many processed patients, we only present here result images of one of them.

First step consists of the affine registration of the atlas and the patient images. As initialization this algorithm needs only a contour description of the brain that it is actually automatically extracted. The global registration is done in a few seconds.

After that, the non-rigid matching should be applied. But demons algorithm is sensible to image intensities, that means that if there is no correspondence beetween intensity the algorithm fails. So, at this point an automatic fitting of atlas and patient histograms is done in order to obtain a linear intensity correction of atlas intensity.

Now, MR atlas is the deformable model and patient images are the reference images. Three parameters have to 


\begin{tabular}{c||c|c||c|c}
\hline \multicolumn{1}{c||}{} & \multicolumn{2}{c||}{ Before extraction } & \multicolumn{2}{c}{ After extraction } \\
\hline \hline Patient & G.M. & W.M. & G.M. & W.M. \\
\hline \hline 1 & 544 & 579.9 & 457.9 & 532.32 \\
2 & 549 & 511 & 470.8 & 453.669 \\
3 & 652.76 & 595.3 & 550.65 & 543.574 \\
4 & 642 & 534 & 543.3 & 485.9 \\
5 & 521.6 & 516.9 & 450.93 & 443.5 \\
6 & 603 & 480 & 520.2 & 413.644 \\
7 & 559.1 & 506.4 & 488 & 460.7 \\
8 & 584 & 487 & 492.75 & 438.58
\end{tabular}

Table 1. Gray and white matter volumes in $\mathrm{mm}^{3}$.

be determined to run the demons algorithm: rigidity of the model, number of scales, and number of iterations at finest scale. For our image dimensions we found the following parameters lead in appropiate results: $\sigma=5$ voxels, 4 scales and 16 iterations at finest scale. The non-rigid registration was executed on a PC-Linux, Pentium III at $700 \mathrm{MHz}$ and computation time was about 30 minutes.

Once the non-rigid transformation is found, it is applied on the label map and a mask of the objects of interest (the cerebral trunk and the cerebellum) is extracted.

In Figure 2 we can see the results for one patient. Axial, sagittal and coronal view are shown and two contours are visualized. One contour (in gray color) corresponds to the SPL atlas contour deformed by the affine transformation. The other one (in white color) is the contour of the registered atlas. In these images we can see that a good matching has been obtained even in the areas where large displacements were needed.

Finally, the calculation of the WM and GM volumes is done by the Shroeter algorithm [6]. In Figure 3 the classification results are shown. The volume computation is done before and after cerebral trunk and cerebellum extraction. The validation of the method has been done in 15 patients but only results of 8 are shown in Table1.

\section{CONCLUSION}

We have implemented a tandem of affine and non-rigid matching techniques for the automatic segmentation of internal brain structures. We have found that the affine registration leads as a better initialization for demons algortihm than a rigid transformation (only 3 rotations and 3 translations). The non-rigid algoritm used here is improved by an automatic fitting of image intensities. We have visually validated that the non-rigid technique reaches a good deformation even when large difference are present but always preserving anatomical differences. These results demonstrate that this combination of techniques is successful for segmentation. We apply this methodology to the automatic ex- traction of the cerebral trunk and the cerebellum to recalculate GM and WM volumes. This will help doctors in the diagnosis of pathologies such as Alzheimer and Parkinson diseases.

\section{ACKNOWLEDGMENT}

The authors wish to thank Dr Ron Kikinis who has provided us with the Surgical Planning Laboratory (SPL) atlas.

\section{REFERENCES}

[1] R. Bajcsy and S. Kovacic, "Multiresolution elastic matching," Computer Vision, Graphics and Image Processing, pp. 1-21, 1989.

[2] G.E. Christensen, R.D. Rabbitt, and M.I. Miller, "3d brain mapping using a deformable neuroanatomy," Physics in Medecine and Biology, pp. 609-618, 1994.

[3] M. Bro-Nielsen and C. Gramkow, "Fast fluid registration of medical images," in Proc Visualization Biomedical Computing Conf. (Lecture Notes in Computer Science), K.H. Hone and R. Kikinis, Eds. 1996, vol. 1131, pp. 267-276, Springer-Verlag.

[4] J.-P. Thirion, "Image matching as a diffusion process: an analogy with maxwell's demons," Medical Image Analysis, vol. 2, no. 3, pp. 243-260, 1998.

[5] S.L. Hartmann, M.H. Parks, P.R. Martin, and B.M. Dawant, "Automatic 3-d segmentation of internal structures of the head in mr images using a combination of similarity and free-form transformations: Part i, methodology and validation on normal subjects," IEEE Transactions on Medical Imaging, vol. 18, no. 10, 1999.

[6] P. Schroeter, J.-M. Vesin, T. Langenberger, and R. Meuli, "Robust parameter estimation of intensity distributions for brain magnetic resonance images," IEEE Transactions on Medical Imaging, vol. 17, no. 2, pp. 172-186, 1998.

[7] R. Kikinis et al, "A digital brain atlas for surgical planning, model driven segmentation and teaching," IEEE Transactions on Visualization and Computer Graphics, vol. 2, no. 3, 1996, http://splweb.bwh.harvard.edu:8000.

[8] O. Cuisenaire, J.P. Thiran, B. Macq, C. Michel, A. De Volder, and F. Marques, "Automatic registration of $3 \mathrm{~d}$ images with a computerized brain atlas," SPIE Medical Imaging, vol. 1719, pp. 438-449, 1996.

[9] B.P.K. Horn and B.G. Schunck, "Determining optical flow," Artificial Intelligence, pp. 185-203, 1981. 